\title{
NOUVELLe
}

\section{Maintien de la perfusion corticale par la sphingosine 1-phosphate lors de la phase aiguë de l'accident vasculaire cérébral ischémique}

Anja Nitzsche ${ }^{1}$, Marine Poittevin ${ }^{1,2}$, Ammar Benarab $^{1}$, Philippe Bonnin ${ }^{3,4}$, Eric Camerer ${ }^{1}$
'Université de Paris,

Paris-Centre de recherche cardiovasculaire (PARCC), Inserm U970, 56 rue Leblanc, 75015 Paris, France.

${ }^{2}$ Institut des vaisseaux et du sang, hôpital Lariboisière, 75010 Paris, France.

${ }^{3}$ Université de Paris, Inserm U1148, hôpital Bichat, 75018 Paris, France.

${ }^{4}$ Université de Paris, APHP, Physiologie clinique-explorations fonctionnelles, hôpital Lariboisière, 75010 Paris France. philippe.bonnin@aphp.fr eric.camerer@inserm.fr
> L'accident vasculaire cérébral ischémique est une cause majeure de morbidité et de mortalité dans le monde; il entraîne un coût économique et sociétal important, qui impose la recherche de stratégies thérapeutiques pour limiter sa gravité. Le traitement en urgence demeure principalement la recanalisation artérielle, soit par thrombolyse pharmacologique, soit par thrombectomie mécanique, qui n'apportent un bénéfice significatif que chez un tiers des patients [1]. Les traitements dits neuro-protecteurs n'ont pas encore montré de bénéfice clair, et la recherche de traitements adjuvants à la recanalisation dénués d'effet indésirable est donc nécessaire. L'ischémie cérébrale est provoquée par l'occlusion d'une artère cérébrale principale par un mécanisme d'embolie d'origine artérielle ou cardiaque, ou par un mécanisme de thrombose artérielle in situ. L'occlusion provoque la formation d'un corps de nécrose juste en aval de l'occlusion artérielle, entouré d'une couronne de tissu cérébral en souffrance ischémique, ou zone de pénombre (Figure 1). L'extension secondaire de la nécrose cérébrale à la zone de pénombre peut être limitée, grâce à la restauration rapide de la perfusion cérébrale par le truchement de la recanalisation rapide ou par la mise en place précoce d'une circulation collatérale contournant l'obstacle [2]. Cette collatéralité peut s'établir par l'intermédiaire d'anastomoses corticales préexistantes, étendues en surface des hémisphères cérébraux entre les territoires vasculaires cérébraux adjacents; la rapidité de sa mise en place conditionne la sauvegarde de la zone de pénombre [3]. L'efficacité de la collatéralité corticale dépend de l'intégrité des circuits micro-vasculaires, de leur perméabilité et de leur capacité à se dilater afin d'assurer l'apport sanguin. Les stratégies visant à améliorer la fonction micro-vasculaire consisteraient alors à inhiber la thrombose d'origine inflammatoire induite par les lymphocytes, à stimuler la fonction endothéliale (vasodilatation fluxdépendante), et à renforcer la barrière hémato-encéphalique dans les territoires atteints $[4,5]$.

La sphingosine 1-phosphate (SIP) est un lipide de signalisation qui joue un rôle majeur dans les systèmes immunitaire et vasculaire. Elle se lie à cinq récepteurs couplés aux protéines $G$ : SIPRI à SIPR5 [6]. La sortie des lymphocytes des organes lymphoïdes dépend de la détection de la sphingosine-1-phosphate (SIP) par le récepteur SIPRI ; l'inhibition des récepteurs SlPRl lymphocytaires entraîne d'ailleurs une lymphopénie profonde. Le récepteur SIPRI est aussi fortement exprimé sur les cellules endothéliales, et la délétion, restreinte aux seules cellules endothéliales, du gène codant ce récepteur chez la souris perturbe l'angiogenèse embryonnaire et postnatale, l'intégrité vasculaire, et la fonction endothéliale [6]. La perte de la signalisation par les récepteurs SIPRI endothéliaux déstabilise les jonctions intercellulaires endothéliales, réduit l'activité de l'enzyme de synthèse du monoxyde d'azote (NO) endothéliale, responsable de la vasoréactivité, et augmente l'expression des molécules d'adhérence des leucocytes [6]. SIPRI joue ainsi un rôle essentiel pour soutenir les fonctions caractéristiques de l'endothélium vasculaire. La SIP est abondante dans le sang circulant, où elle est associée principalement aux lipoprotéines de haute densité et à l'albumine. Les érythrocytes et les cellules endothéliales sont des sources majeures de SIP à l'état stable; les plaquettes le sont également, mais la libération de la SIP par les plaquettes est subordonnée à leur activation [6]. L'endothélium des vaisseaux capillaires cérébraux présente une particularité, constitutive de la barrière hémato-encéphalique: les cellules endothéliales sont unies par des jonctions intercellulaires serrées ne laissant diffuser que les molécules de petite masse moléculaire. Cette barrière joue donc un rôle protecteur du tissu cérébral, mais empêche l'extravasation de la SIP circulante.

Le fingolimod est un immunomodulateur utilisé dans le traitement de la sclérose en plaques. Phosphorylé par les kinases de sphingosine, le fingolimod peut agir 
A Fonction endothéliale normale, mise en place de la collatéralité artérielle corticale

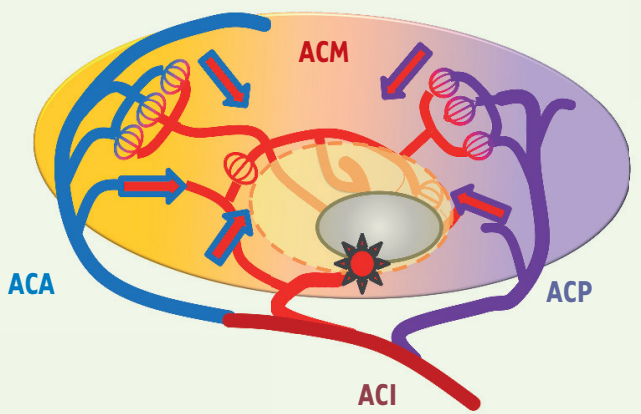

B Déficience du récepteur SIPRI sur les cellules endothéliales, absence de mise en place de la collatéralité corticale

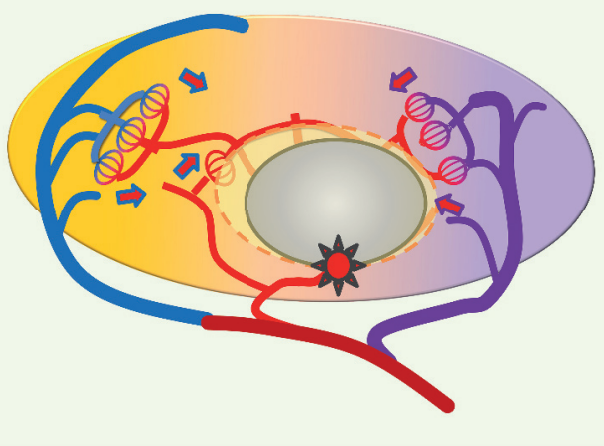

C Administration d'un agoniste du récepteur SIPRI dans les 6 heures après le début de l'ischémie, stimulation de la collatéralité corticale

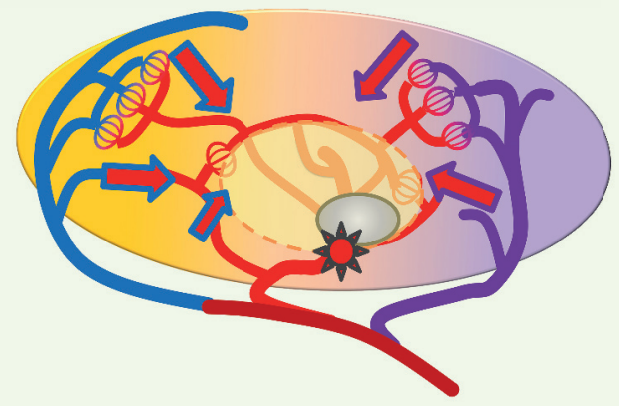

Occlusion de l'artère cérébrale moyenne

Zone de pénombre initiale

Corps de nécrose résultant (à 6 heures)

ACA : artère cérébrale antérieure

$A C P$ : artère cérébrale postérieure

$A C M$ : artère cérébrale moyenne

$\mathrm{ACl}$ : artère carotide interne

Figure 1. Signalisation par sphingosine-1-phosphate (SIP) au cours de l'accident vasculaire cérébral ischémique expérimental chez la souris. A. Après occlusion de l'artère cérébrale moyenne, la signalisation par la sphingosine 1-phosphate (SIP) promeut l'intégrité des parois vasculaires, la perméabilité des lumières vasculaires, et la mise en place précoce de la collatéralité corticale avec perfusion rétrograde dans la zone de pénombre vers le corps de nécrose, à partir des anastomoses corticales étendues entre les différents territoires vasculaires de chaque artère cérébrale. Cette signalisation s'effectue essentiellement par l'intermédiaire du récepteur SIPRI des cellules endothéliales cérébrales, qui est localisé à la face extravasculaire de ces cellules. Comme la SIP ne traverse pas la barrière hémato-encéphalique, la production locale de SIP par les cellules endothéliales est nécessaire à l'obtention des effets vasculaires sus-mentionnés. B. En l'absence des fonctions protectrices de la signalisation par la S1P, faisant suite, par exemple, à la délétion du gène codant le récepteur SIPRI dans les cellules endothéliales, le corps de nécrose s'étend rapidement aux dépens de la zone de pénombre, et la lésion cérébrale résultante est étendue. $C$. En stimulant la signalisation de la SIP dans les cellules endothéliales cérébrales par des agonistes pharmacologiques ciblant le récepteur SIPRI, l'extension de la nécrose est contrecarrée, à condition toutefois que le passage de la barrière hémato-encéphalique par ces agonistes s'effectue dans un délai court après le début de l'ischémie (figure adaptée de [8]).

comme un agoniste de plusieurs récepteurs SIPR. Dans les lymphocytes, cet effet agoniste induit une internalisation rapide de SIPRl, qui les désensibilise à la SIP. Le fingolimod a donné des résultats prometteurs lorsqu'il a été utilisé pour traiter l'accident vasculaire cérébral d'origine ischémique ou hémorragique dans des modèles expérimentaux et dans des essais cliniques à petits effectifs [4, 7]. D'autres modulateurs spécifiques du récepteur
SIPRl présentent une action bénéfique similaire, suggérant que c'est bien ce récepteur qui est la cible du fingolimod dans ce contexte. Jusqu'à présent, tous ces médicaments étaient considérés comme protecteurs principalement par la modulation de l'immunité pendant l'ischémie cérébrale [4]. Cependant, considérant le rôle majeur du récepteur SIPRl dans le maintien des fonctions endothéliales dans les autres organes, les mécanismes d'action du récepteur
SIPRl et de ses modulateurs au cours de l'ischémie cérébrale ont été analysés à l'aide de différents outils génétiques et expérimentaux [8].

Chez la souris soumise à une ischémie cérébrale transitoire ou permanente, un déficit en récepteur SIPRI restreint aux cellules hématopoïétiques n'a entraîné que des effets minimes sur le volume de la lésion cérébrale, alors qu'un déficit de ce récepteur restreint aux cellules endothéliales a provoqué une extension 
de l'ischémie, montrant bien l'implication bénéfique de ce récepteur dans le cerveau [8]. Le déficit de signalisation du récepteur SIPRI restreint aux cellules endothéliales était également associé à une augmentation de l'expression des molécules d'adhérence des leucocytes, à une altération de la mise en place de la collatéralité corticale vers la zone de pénombre, à une réduction de la perfusion des petits vaisseaux, et à l'apparition d'un œdème cérébral (Figure 1). Malgré une disposition vasculaire normale, en particulier des anastomoses corticales, le dysfonctionnement endothélial chez ces souris était accompagné d'une expansion rapide du corps de nécrose dès la phase précoce, avec doublement de la taille de l'infarctus cérébral par rapport aux souris témoins. Étonnamment, l'inactivation des kinases responsables de la synthèse plasmatique de la SIP dans les cellules sanguines circulantes n'a pas eu d'impact sur le volume de lésion cérébrale, alors que leur inactivation dans les cellules endothéliales a reproduit le déficit induit par l'absence de récepteur SIPRI à la surface des cellules endothéliales. Comme la SIP circulante ne passe pas la barrière hémato-encéphalique, et comme les récepteurs SIPRI sont principalement situés à la face extravasculaire de la cellule endothéliale cérébrale, l'activation de ces récepteurs par leur ligand, la SIP, nécessite une production de ce ligand par les cellules endothéliales ellesmêmes, une situation contrastant avec celle de l'activation de ces récepteurs au cours du développement du système vasculaire ou dans l'homéostasie circulatoire, qui dépend alors de la liaison de ces récepteurs avec la SIP circulante [6, 9 , 10]. À l'appui de ce modèle, seuls les agonistes du récepteur SIPRI pénétrant la barrière hémato-encéphalique ont eu la capacité de protéger les souris de l'ischémie cérébrale dans les modèles d'accident vasculaire cérébral, capacité perdue chez la souris mutante dont le récepteur SIPRI est supprimé sélectivement dans les cellules endothéliales [8]. La production de la SIP par la cellule endothéliale cérébrale et sa signalisation par son récepteur SIPRI sont donc nécessaires à la régulation des fonctions de la cellule endothéliale au cours de I'ischémie cérébrale. Bien que cette signalisation ne soit que partiellement soutenue par l'autoproduction de SIP, elle peut être stimulée par des agonistes pharmacologiques pénétrant la barrière hémato-encéphalique pour favoriser localement le rétablissement de la circulation sanguine de façon indépendante de l'immunosuppression induite par certains modulateurs de SIPRI (Figure 1). A la lumière de l'utilisation des modulateurs de SIPRI pour traiter la sclérose en plaques, et compte tenu des résultats des essais cliniques effectués à petite échelle chez des patients victimes d'accidents vasculaires cérébraux, on sait que la modulation de SIPRl est peu susceptible d'être associée à des complications hémorragiques, même lorsque ces médicaments sont associés à des thrombolytiques [7, 11]. Une stratégie thérapeutique utilisant des agonistes adaptés pour cibler SIPRI sur les cellules endothéliales pourrait donc être envisagée chez les patients dès le diagnostic d'accident vasculaire cérébral, sans attendre le résultat de la recanalisation. Le rôle protecteur majeur du récepteur SIPRI endothélial dans l'homéostasie vasculaire et au cours de l'ischémie cérébrale, et les stratégies thérapeutiques visant à son recrutement pourraient également être pertinents pour la prise en charge au cours de l'accident vasculaire cérébral hémorragique, de démence vasculaire, ou d'accidents ischémiques dans d'autres organes. $\diamond$

Endothelial sphingosine 1 -phosphate signaling maintains perfusion of the cerebral cortex in ischemic stroke

\section{LIENS D'INTÉRÊT}

Les auteurs déclarent n'avoir aucun lien d'intérêt concernant les données publiées dans cet article.

\section{RÉFÉRENCES}

1. Jauch EC, Saver JL, Adams HP Jr, et al. Guidelines for the early management of patients with acute ischemic stroke: a guideline for healthcare professionals from the American heart association/American stroke association. Stroke $2013 ; 44: 870-947$.

2. Manning NW, Campbell BC, Oxley TJ, Chapot R. Acute ischemic stroke: time, penumbra, and reperfusion. Stroke $2014 ; 45: 640-4$.

3. Bonnin P, Mazighi M, Charriaut-Marlangue C, Kubis N. Early collateral recruitment after stroke in infants and adults. Stroke $2019 ; 50: 2604-11$.

4. Dreikorn M, Milacic Z, Pavlovic V, et al. Immunotherapy of experimental and human stroke with agents approved for multiple sclerosis: a systematic review. Ther Adv Neurol Disord 2018 ; 11 : 1-14.

5. Shuaib A, Butcher K, Mohammad AA, et al. Collateral blood vessels in acute ischaemic stroke: a potential therapeutic target. Lancet Neurol 2011 ; 10: 909-21

6. Proia RL, Hla T. Emerging biology of sphingosine-1phosphate: its role in pathogenesis and therapy. J Clin Invest 2015 ; 125 : 1379-87.

7. Tian DC, Shi K, Zhu Z, et al. Fingolimod enhances the efficacy of delayed alteplase administration in acute ischemic stroke by promoting anterograde reperfusion and retrograde collateral flow. Ann Neurol 2018 ; 84 : 717-28.

8. Nitzsche A, Poittevin M, Benarab A, et al. Endothelial S1Pl signaling counteracts infarct expansion in ischemic stroke. Circ Res $2021 ; 128: 363-82$.

9. Camerer $\varepsilon$, Regard JB, Cornelissen I, et al. Sphingosine-1-phosphate in the plasma compartment regulates basal and inflammation-induced vascular leak in mice. J Clin Invest 2009 ; 119 : 1871-9.

10. Gazit SL, Mariko B, Therond P, et al. Platelet and erythrocyte sources of SIP are redundant for vascular development and homeostasis, but both rendered essential after plasma SIP depletion in anaphylactic shock. Circ Res $2016 ; 119$ : el10-26.

11. Li YJ, Shi SX, Liu Q, et al. Targeted role for sphingosinel-phosphate receptor $l$ in cerebrovascular integrity and inflammation during acute ischemic stroke. Neurosci Lett $2020 ; 735$ : 135160.

\section{Retrouvez toutes les Actualités de la Myologie sur les sites de :}
la Société Française de Myologie
www.sfmyologie.org
sfm
la filière de santé neuromusculaire FILNEMUS
www.filnemus.fr

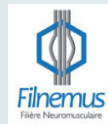

